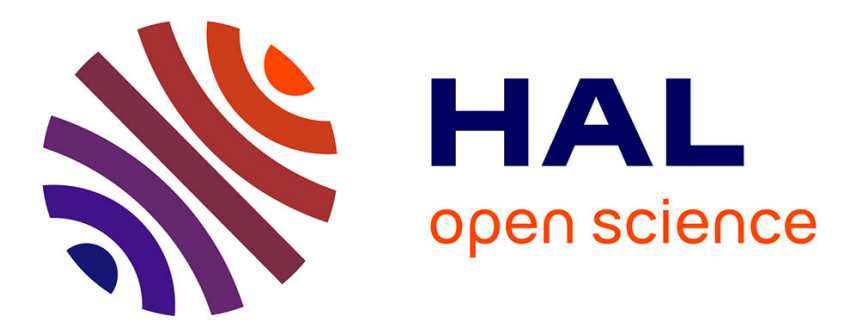

\title{
Detection of single photoluminescent diamond nanoparticles in cell and study of the internalization pathway
}

Orestis Faklaris, Damien Garrot, Vandana Joshi, Frédéric Druon, Jean-Paul P Boudou, Thierry Sauvage, Patrick Georges, Patrick Curmi, François Treussart

\section{To cite this version:}

Orestis Faklaris, Damien Garrot, Vandana Joshi, Frédéric Druon, Jean-Paul P Boudou, et al.. Detection of single photoluminescent diamond nanoparticles in cell and study of the internalization pathway. Small, 2008, 4 (12), pp.2236-2239. 10.1002/smll.200800655 . hal-02898875

\section{HAL Id: hal-02898875 https://hal.science/hal-02898875}

Submitted on 14 Jul 2020

HAL is a multi-disciplinary open access archive for the deposit and dissemination of scientific research documents, whether they are published or not. The documents may come from teaching and research institutions in France or abroad, or from public or private research centers.
L'archive ouverte pluridisciplinaire HAL, est destinée au dépôt et à la diffusion de documents scientifiques de niveau recherche, publiés ou non, émanant des établissements d'enseignement et de recherche français ou étrangers, des laboratoires publics ou privés. 


\section{stmall}

DOI: $10.1002 /$ smll.200800655

\section{Detection of single photoluminescent diamond nanoparticles in cell and study of the internalization pathway}

Orestis Faklaris, Damien Garrot, Vandana Joshi, Frédéric Druon, Jean-Paul Boudou, Thierry Sauvage, Patrick Georges, Patrick Curmi, and François Treussart*

[*] Prof. F. Treussart, O. Faklaris, Dr. D. Garrot

Laboratoire de Photonique Quantique et Moléculaire,

UMR CNRS 8537, Cachan (France)

E-mail: francois.treussart@ens-cachan.fr

Dr. V. Joshi, Dr. P. Curmi

Laboratoire Structure et Activité des Biomolécules Normales et Pathologiques,

Université Evry-Val-d'Essonne and INSERM U829, Evry (France)

Dr. F. Druon, Dr. P. Georges

Laboratoire Charles Fabry de l'Institut d'Optique,

UMR CNRS 8501, Palaiseau (France)

Dr. J.P. Boudou

Laboratoire BioEmCo, Université Pierre et Marie Curie,

UMR CNRS 7618, Paris (France)

Dr. T. Sauvage

Conditions Extrêmes et Matériaux : Haute Température et Irradiation,

UPR CNRS 3079, Orléans, (France)

Supporting Information is available on the WWW under http://www.small-journal.com or from the author.

Keywords: diamond, nanoparticles, photoluminescence, biomarker, cytotoxicity

Diamond nanoparticles are promising photoluminescent probes for tracking intracellular processes, due to embedded perfectly photostable color centers. In this work, we investigate the spontaneous internalization of such nanoparticles (diameter $25 \mathrm{~nm}$ ) in HeLa cells by confocal microscopy and time-resolved techniques. Nanoparticles are observed inside the cell cytoplasm at the single particle and single color center level, assessed by time correlation intensity measurements. Improvement of the nanoparticles signal-to-noise ratio inside cell is achieved using a pulsed excitation laser and a time resolved detection taking advantage of the long radiative lifetime of the color center excited state, compared to cell auto-fluorescence. The internalization pathways are also investigated, with endosomal marking and colocalization analyses. The low colocalization ratio observed proves that nanodiamonds are not trapped in endosomes, a promising result in prospect of drug delivery by these nanoparticles. We also prove a low cytotoxicity of these nanoparticles in this cell line. 


\section{small}

\section{Introduction}

Single molecule observation is of great importance in life sciences. Individual probing enables studies of biomolecules interactions directly. In order to achieve an efficient biolabeling, a reliable marker is needed. Organic dyes and fluorophores are widely used, but they lack of photophysical stability. An alternative recently introduced are semiconductor nanocrystals. ${ }^{[1]}$ Although they are very efficient for multicolour staining and have a much lower photobleaching yield than dyes, they suffer from blinking and may be cytotoxic in long term. ${ }^{[2]}$ In contrast, diamond nanoparticles (NDs) containing nitrogen-vacancy color centers $\left(\mathrm{NV}{ }^{[3]}\right.$ represent a promising alternative in the family of inorganic photoluminescent biomarkers. ${ }^{[4,5]}$ Indeed NV color centers neither photobleach nor blink, which allows reliable single particle tracking.${ }^{[6,7]}$ NDs biocompatibility was demonstrated for Ib type nanodiamonds ${ }^{[4,5]}$, as well as for detonation NDs which present an outer $\mathrm{sp}^{2}$-hybridized carbon-shell. ${ }^{[8,9,10]}$ Thus, such photoluminescent nanodiamonds (PNDs) posses the potential to be used as long term traceable delivery vehicles for the translocation and tracking of biomolecules into cells. In such applications the size of the photoluminescent nanodiamonds has to be as small as possible, i.e. at least under $50 \mathrm{~nm}$, to reduce the impact of the nanoparticles on the natural behavior of the grafted molecule. One must be still able to detect the signal of such tiny material over the cellular background.

In this work, we demonstrate the efficient uptake of PNDs of less than $50 \mathrm{~nm}$ in size at the single particle level in cultured cells. The photoluminescence properties of individual nanoparticles in cells were studied by a home-made confocal microscope equipped with a single photon detector, a time correlation measurement setup and an imaging spectrograph. Furthermore we increased the signal (from PNDs)-to-noise ratio by selecting only the late photons, after pulse excitation of the sample, taking advantage of the rather long radiative lifetime of NV color centers ( $\approx 20 \mathrm{~ns}$ ). Interestingly we observed, using combined 


\section{smallion}

immunocytochemistry, that about $20 \%$ of the internalized PNDs colocalize with endosomes which implies that a significant portion of these particles does not stay trapped in the endosomes, which is a critical point for biomolecules vectorization applications. Finally, cell viability assays confirmed the good biocompatibility of these innovative biomarkers.

\section{Results and discussion}

\subsection{Detection of individual PND in cultured cells}

Presence of internalized PNDs into the cells is demonstrated on confocal scans obtained at different heights along the $z$-axis, using $\mathrm{cw}$ laser excitation at $488 \mathrm{~nm}$ (see Supporting Information Figure 2). We are able to discriminate single particles from agglomerated NDs. As shown in Figure 1, the photoluminescence spot of the PND has a diffraction limited size $(\mathrm{FWHM}=250 \mathrm{~nm}$ ). Photoluminescence spectrum from a single spot allows us to confirm that emission originates from $\mathrm{NV}$ color centers. Intensity time correlation measurement of emitted light yields a photon antibunching corresponding to two emitters inside the diamond nanoparticle under study. In a recent study we have shown, using the same nanodiamond sample (of size $<50 \mathrm{~nm}$ ) than the one considered in the present work, that about one third of the $20 \mathrm{~nm}$ NDs population contains 1 to $2 \mathrm{NV}$ centers while the remaining does not contain any NV center ${ }^{[12]}$ In the present study, roughly $40 \%$ of the NDs that can be observed by photoluminescence inside the cell contain less than 4 emitters, which is a further indication that the nanoparticles detected are either two $25 \mathrm{~nm}$ particles agglomerates or more probably single (isolated and not agglomerated) nanoparticles with largest particle size, up to $50 \mathrm{~nm}$. This observation is important for future applications such as drug delivery, which require a high mobility of PNDs inside the cell.

\subsection{Time-gated imaging of internalized PNDs}

We can take advantage of the "long" fluorescence lifetime of NV centers in PNDs to enhance image contrast and sensitivity of PNDs detection in cultured cells. Despite the fact that the fluorescence lifetime of NV centers in PNDs presents an important dispersion due to the 


\section{smallion}

different crystalline environments, most of the NV centers have a lifetime greater than 15 ns. ${ }^{[13,14]}$ Time selection of "late" photons only, ensures that the signal is dominated by the NV color center emission. A similar technique has been used for quantum dots. ${ }^{[15]}$ The photoluminescence decay time is obtained by using a time-to-amplitude converter (TAC). The start and the stop pulses are provided by the avalanche photodiode and a signal synchronized with the laser pulses respectively. A PC plug-in multi-channel analyzer board acquires the signal from the TAC and produces histograms of photon arrival times for each pixel in the raster scan. Figure 2 shows time-resolved confocal images of a HeLa cell after incubation with PNDs. Selection of "late" photons reduces dramatically the noise from the autofluorescence of the cell and in turn enhances the PNDs signal-to-background ratio by an order of magnitude (Figure 2(b)). The high contrast and sensitivity combined with the perfect photostability allow a precise localization of the nanoparticle inside the cell.

\subsection{Nanodiamonds uptaken by cells only partly colocalize with endosomes}

A rather low degree of colocalization was observed between early endosomes and the PNDs signal as shown on Figure 3. Out of 20 cells and 256 internalized PNDs for an incubation time of 2 hours, a colocalization was observed for about $21 \%$ of the particles with an error margin of 6\%. Two reasons can explain this result: either the PNDs do not enter the cells by endocytosis or they are early liberated from the endosomes in the cytosol or in the lysosomes. The fact that the NDs do not stay inside the endosomes is a positive indication that PNDs are suitable for applications in drug delivery. Further investigations are in progress to understand the full path of the PNDs journey into the cell.

\subsection{Nanodiamonds are well tolerated by cell in culture} Presence of putative cytotoxicity of PNDs was investigated with the MTT test. HeLa cells were precultured for 24 hours before the addition of PNDs at different concentrations (5.7$480 \mu \mathrm{g} / \mathrm{ml}$ ) in $1 \mathrm{ml}$ of culture medium. The culture was carried out for $48 \mathrm{~h}$. Results show 


\section{stamestismall}

that nanodiamonds do not significally alter the viability of the cells except at the highest nanodiamond concentration (Figure 4), which is however much higher than what would be required for drug delivery applications.

\section{Conclusions}

In conclusion, we show here that nanodiamonds are internalized by HeLa cells in culture, and have a low toxicity over a period of $48 \mathrm{~h}$. The PNDs are easily detected at the single particle level over the cell autofluorescence background by time-gated imaging. We also show for the first time that nanodiamonds are mainly dispersed in extra-endosomal region, after an incubation time of 2 hours. This result indicates that this material may be a good candidate for biomolecules delivery and long term tracking.

\section{Experimental Section}

PND preparation : PNDs are prepared following the procedure described in Ref. [12].

Briefly, the starting material is synthetic type Ib diamond powder (SYP 0-0.05, Van Moppes, Geneva) with a specified size smaller than $50 \mathrm{~nm}$, that we further select in size by centrifugation. Creation and stabilization of NV centers is performed by high energy proton irradiation and subsequent annealing. Deagglomeration of PNDs is achieved by strong acid treatment $\left(\mathrm{H}_{2} \mathrm{SO}_{4}: \mathrm{HNO}_{3}, 1: 1 \mathrm{vol}\right.$ : vol, at $80^{\circ} \mathrm{C}$ for $\left.24 \mathrm{~h}\right)$. This acid treatment also allowed us to obtain a stable suspension of NDs smaller than $50 \mathrm{~nm}$ (see Supporting Information Figure 1 for Atomic Force Microscope and Dynamic Light Scattering measurements) in deionised water thanks to repulsive electrostatic interactions between charged surface chemical groups, mainly carboxylic groups. ${ }^{[5]}$

Imaging PND in cells : Distribution of PNDs in fixed cells is assayed using a home-made scanning stage confocal microscope. Laser excitation of PNDs photoluminescence is done either with a continuous-wave (cw) optically pumped semiconductor laser emitting at a wavelength of $488 \mathrm{~nm}$, or with a home-made picosecond-pulse laser relying on a diode 


\section{stmall}

pumped Nd: $\mathrm{YVO}_{4}$ passively mode-locked emitting at $1064 \mathrm{~nm}$ (repetition rate $5 \mathrm{MHz}$, pulse duration $20 \mathrm{ps}{ }^{[16,17]}$ ) subsequently doubled in frequency to get $532 \mathrm{~nm}$ excitation. The excitation beam is focused onto the sample with a high-numerical aperture oil immersion microscope objective ( $\times 60,1.4$ numerical aperture). Photoluminescence, collected from the sample by the same objective, is spectrally filtered by a high-pass filter (either RazorEdge LP02-514RU-25, from Semrock, USA, 97\% transmission between 521-1160 nm when we use the cw laser at $488 \mathrm{~nm}$; or RazorEdge LP03-532RU-25, 97\% transmission between 539$1200 \mathrm{~nm}$ for pulsed excitation at $532 \mathrm{~nm}$ ) and spatially filtered by a $30 \mu \mathrm{m}$ diameter pinhole. It is then detected by an avalanche photodiode in the single photon counting mode (SPCMAQR14; Perkin-Elmer). The number of NV color centers per nanoparticle is measured using time correlation of intensity experiments, relying on a Hanbury Brown and Twiss correlator. ${ }^{[1]}$

PND uptake by cell and immunofluorescence analysis: HeLa cells were grown in standard conditions on glass coverslips in DMEM culture medium supplemented with $10 \%$ FCS. To study internalization of PNDs, cells were seeded at a density of $10^{5}$ cells $/ 1.3 \mathrm{~cm}^{2}$ and grown at $37^{\circ} \mathrm{C}$ in a humidified incubator under $5 \% \mathrm{CO}_{2}$ atmosphere. $24 \mathrm{~h}$ after cell seeding, different quantities of PNDs aqueous suspensions were added to the cell medium. The cells were grown under the same conditions for an additional period of time $(2 \mathrm{~h}$ for fluorescence detection and up to $48 \mathrm{~h}$ for viability MTT test). After incubation, the excess of PNDs was removed by washing cells with PBS. Cells were then fixed with 4\% paraformaldehyde in PBS and mounted on microscope slides for phase contrast and confocal imaging. For immunofluorescence analyses, the endosomes were stained with Fluorescein-conjugated Mouse Anti-human Early Endosome Antigen (EEA) (BD Transduction Laboratories, USA). 


\section{stimall}

\section{Acknowledgements}

We are grateful to Jean-François Roch for fruitful discussions and help in starting application of nanodiamonds in biology. This work was supported by the European Commission through the project "Nano4Drugs" (contract LSHB-2005-CT-019102), and by Agence Nationale de la Recherche through the project "NaDia" (contract ANR-2007-PNANO-045).

[1] X. Michalet, F. F. Pinaud, L. A. Bentolila, J. M. Tsay, S. Doose, J. J. Li, G. Sundaresan, A. M. Wu, S. S. Gambhir, S. Weiss, Science 2005, 307 (5709), 538-544.

[2] C. Kirchner, T. Liedl, S. Kudera, T. Pellegrino, A. M. Javier, H. E. Gaub, S. Stolzle, N. Fertig, W. Parak, Nano Lett. 2005, 5, 331-338.

[3] A. Gruber, A. Dräbenstedt, C. Tietz, L. Fleury, J. Wrachtrup, C. Von Borczyskowsky, Science 1997, 276, 2012-2014.

[4] S. J. Yu, M. W. Kang, H. C. Chang, K. M. Chen, Y. C. Yu, J. Am. Chem. Soc. 2005, $127,17604-17605$.

[5] C. C. Fu, H. Y. Lee, K. Chen, T. S. Lim, H. Y. Wu, P. K. Lin, P. K. Wei, P. H. Tsao, H. C. Chang, W. Fann, Proc Natl Acad Sci U.S.A. 2007, 104, 727-732.

[6] F. Neugart, A. Zappe, F. Jelezko, C. Tietz, J.P. Boudou, A. Krueger, J. Wrachtrup, Nano Lett. 2007, 7, 3588-3591.

[7] Y.R. Chang, H. Y. Lee, K. Chen, C. C. Chang, D. S. Tsai, C. C. Fu, T.S. Lim, Y. K. Tzeng, C.Y. Fang, C.C. Han, H.C. Chang, W. Fann Nature Nanotech. 2008, 3, 284-288.

[8] A. M. Schrand, L. Dai, J. J. Schlager, S. Hussain, E. Osawa, Diam. Rel. Mat. 2007, $16,2118-2123$.

[9] A. M. Schrand, H. J. Huang, C. Carlson, J. J. Schlager, E. Osawa, S. M. Hussain, L. Dai J. Phys. Chem. B 2007, 16, 2118-2123.

[10] H. Huang, E. Pierstorff, E. Osawa, E. Ho, Nano Lett. 2007, 1, 3305-3314. 


\section{sumant}

[11] F. Treussart, V. Jacques, E Wu, T. Gacoin, P. Grangier, J. F. Roch, Physica B 2006, $376,926-929$.

[12] Y. Sonnefraud, A. Cuche, O. Faklaris, J.P. Boudou, T. Sauvage, J. F. Roch, F.

Treussart, S. Huant, Opt. Lett. 2008, 33, 611-613.

[13] A. Collins, M. Thomaz, M. Jorge, J. Phys. C.: Solid State Phys. 1983, 16, 2177-2181.

[14] A. Beveratos, R. Brouri, T. Gacoin, J. P. Poizat, P. Grangier, Phys. Rev. A 2001, 64, 061802 .

[15] M. Dahan, T. Laurence, F. Pinaud, D. S. Chelma, A. P. Alivisatos, M. Sauer, S. Weiss, Opt. Lett. 2001, 26, 825-827.

[16] C. Gerhard, F. Druon, P. Georges, Optics Express 2006, 14, 7093-7098.

[17] D. Papadopoulos, S. Forget, M. Delaigue, F. Druon, F. Balembois, P. Georges, Opt. Lett. 2003, 28, 1838-1840.

Received:

Revised: Published online on 


\section{sutumests small}

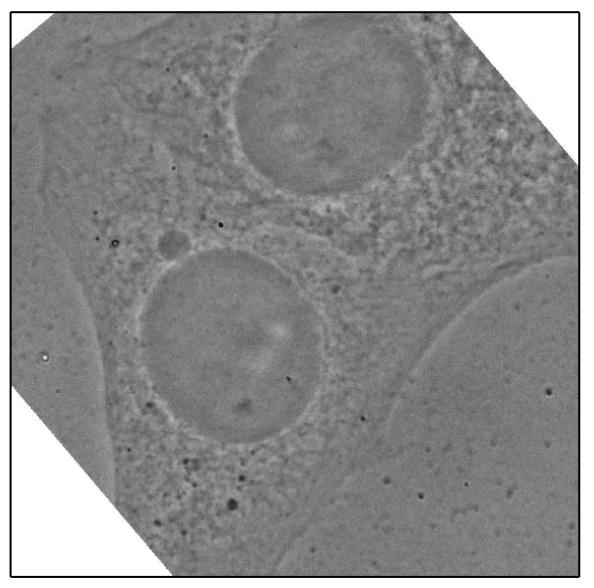

(a)
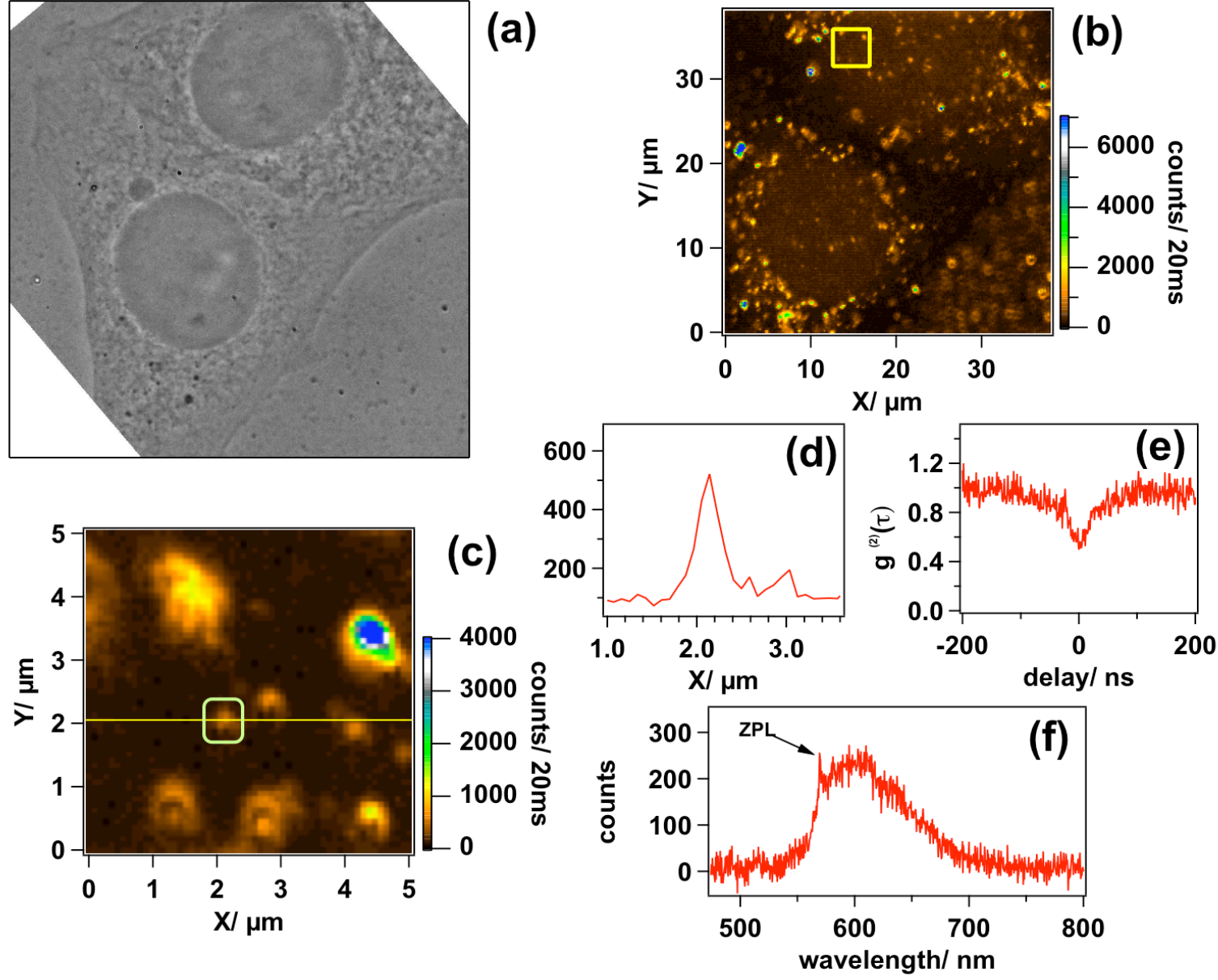

Figure 1: Photoluminescent nanodiamonds (PNDs) in HeLa cells : (a) Phase contrast image showing two cells; (b) Confocal scan at $z=1500 \mathrm{~nm}$ above the coverslip surface, cw excitation laser at wavelength $488 \mathrm{~nm}$, power $0.5 \mathrm{~mW}$; (c) Zoomed confocal scan of the yellow squared region from (b); (d) Intensity profile of the PND encircled in scan (c), FWHM=250 nm; e)

Normalized time correlation of intensity function of the same PND, with photon antibunching at zero delay associated to the emission of $2 \mathrm{NV}$ color centers, excitation wavelength $488 \mathrm{~nm}$, power $2 \mathrm{~mW}$; (f) Photoluminescence spectrum of the same PND corresponding to the emission of the neutral $\mathrm{NV}^{(0)}$ color center, identified thanks to its Zero Phonon Line (ZPL) at $575 \mathrm{~nm} .^{[11]}$ 


\section{small}
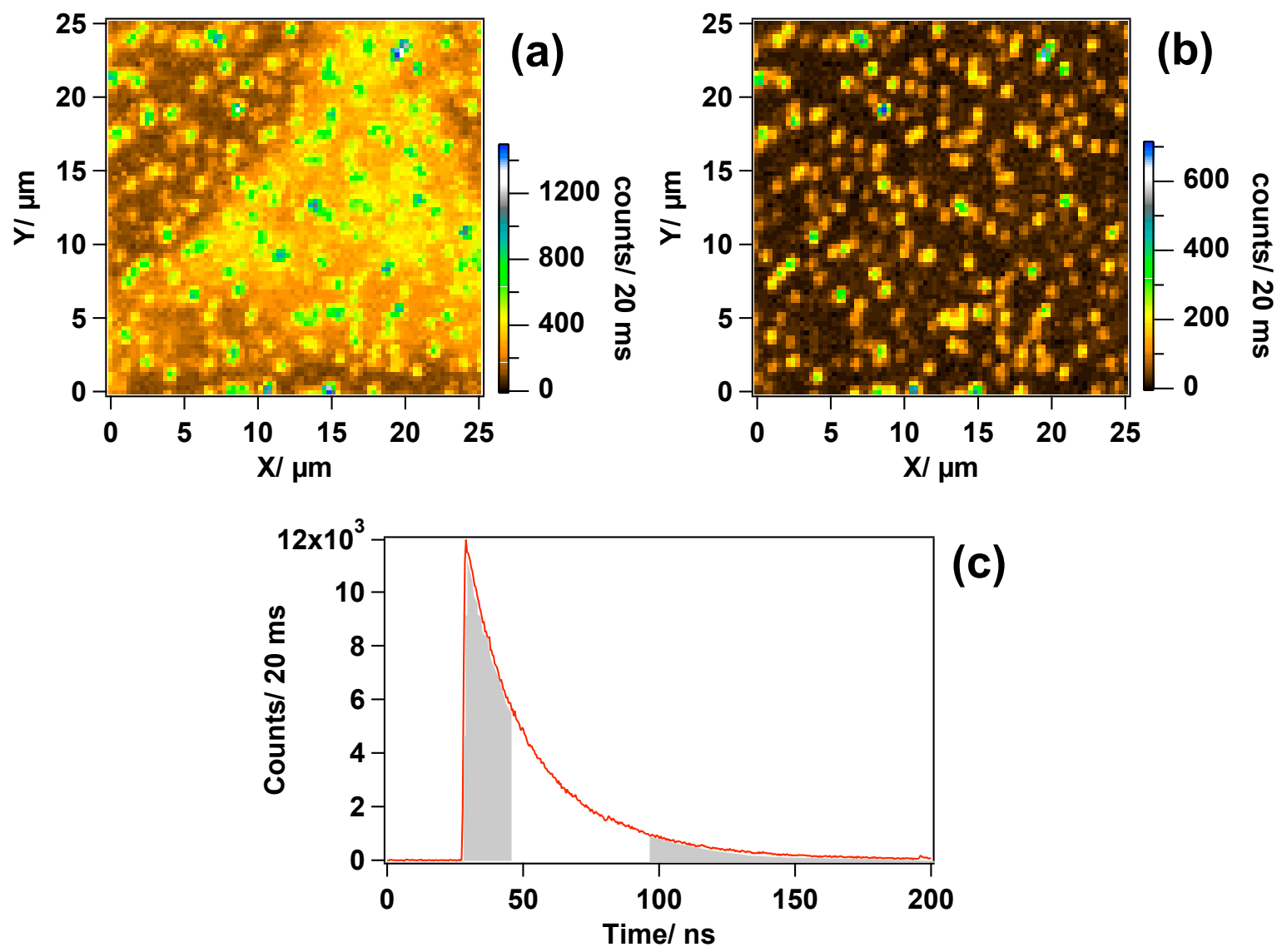

Figure 2: Time-resolved confocal rasterscans of a fixed HeLa cell containing PNDs: (a) rasterscan obtained from all detected photons, displaying PNDs together with autofluorescence from the cell; (b) Time-gated rasterscan constructed from photons detected between 15 and $53 \mathrm{~ns}$ after the laser excitation pulse. Scan area is $25 \times 25 \mu \mathrm{m}$. The mean laser power is $700 \mu \mathrm{W}$ at $532 \mathrm{~nm}$; integration time is $20 \mathrm{~ms} /$ pixel; c) NV color center photoluminescence time decay from one of the nanodiamonds shown in Figure 2 (a), on which we have shown in gray the time-delay range associated to photons excluded to build Figure 2 (b) time-gated rasterscan. 


\section{Submitted to}

(a)

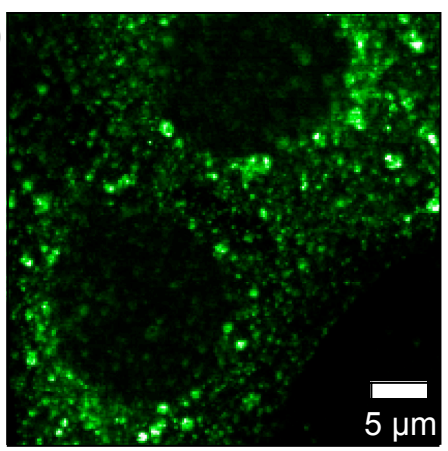

(b)

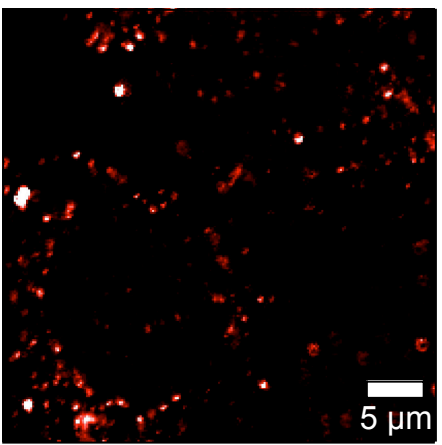

(c)

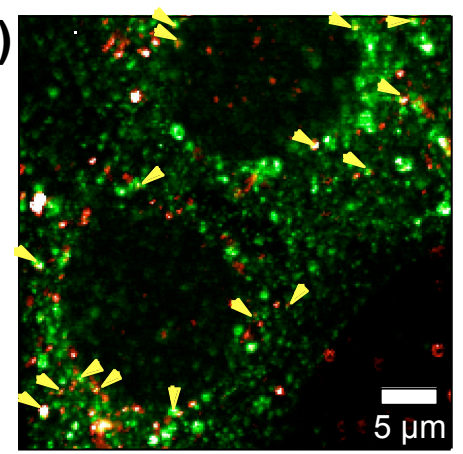

Figure 3: A subset of PNDs co-localized with endosomes. (a) confocal rasterscan showing early endosomes labeled by EEA - FITC conjugate (same cells than the ones of Figure 1 at $z=1500 \mathrm{~nm}$ above coverslip surface); (b) photoluminescence of PNDs ; (c) Merged image, with arrows showing 16 PNDs found to be colocalized with endosomes, out of the 65 photoluminescent nanodiamonds uptaken by these cells.

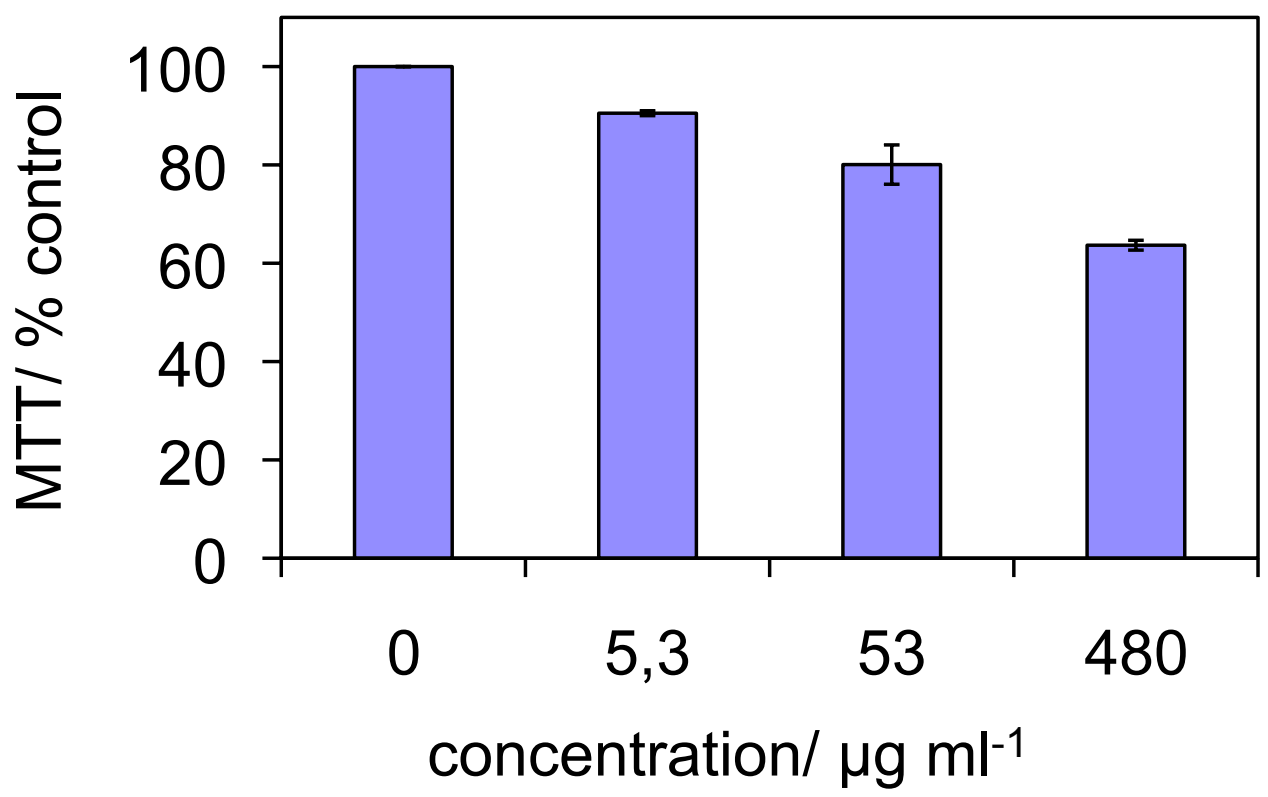

Figure 4: MTT assay results, displaying the proportion of living cells, $48 \mathrm{~h}$ after the addition of aqueous suspension of nanodiamonds at increasing NDs concentrations. 


\section{The table of contents entry}

Diamond nanoparticles are promising photoluminescent probes for tracking intracellular processes, due to embedded perfectly photostable color centers. We investigate the spontaneous internalization process of such nanoparticles (diameter $25 \mathrm{~nm}$ ) in HeLa cells by confocal microscopy and time-resolved techniques. Our preliminary conclusion is that nanodiamonds are not trapped in endosomes (low colocalisation fraction, marked by yellow arrow on the Figure).

TOC Keyword : diamond, nanoparticle, probe, photoluminescence

O. Faklaris, D. Garrot, V. Joshi, F. Druon, J.-P. Boudou, T. Sauvage, P. Georges, P. Curmi, and F. Treussart*

Detection of single photoluminescent diamond nanoparticles in cell and study of the internalization pathway

\section{ToC figure}

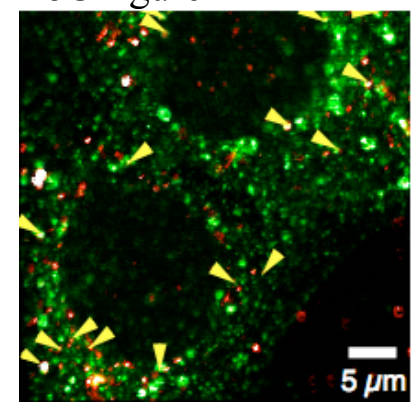

Page Headings

Left page: O. Faklaris et al.

Right page: Internalization pathway of single diamond nanoparticles in cell 